\title{
Nonclassical potential solutions of partial differential equations
}

\author{
GEORGE W. BLUMAN ${ }^{1}$ and ZHENYA YAN ${ }^{2}$ \\ ${ }^{1}$ Department of Mathematics, University of British Columbia, Vancouver, BC, Canada V6T $1 Z 2$ \\ email: bluman@math.ubc.ca \\ ${ }^{2}$ Key Laboratory of Mathematics Mechanization, Institute of Systems Science, AMSS, \\ Chinese Academy of Sciences, Beijing 100080, P. R. China \\ email: zyyan@mmrc.iss.ac.cn
}

(Received 30 July 2003; revised 20 August 2004)

\begin{abstract}
For a given scalar partial differential equation (PDE), a potential variable can be introduced through a conservation law. Such a conservation law yields an equivalent system (potential system) of PDEs with the given dependent variable and the potential variable as its dependent variables. Often there is also another equivalent scalar PDE (potential equation) with the potential variable as its dependent variable. The Nonclassical Method for obtaining solutions of PDEs is a generalization of the Classical Method for obtaining invariant solutions from point symmetries admitted by a given PDE. As a prototypical example, the nonlinear heat conduction equation is used to demonstrate that the Nonclassical Method applied to a potential equation can yield new solutions (nonclassical potential solutions) of a given PDE that are unobtainable as invariant solutions from admitted point symmetries of the given PDE, a related potential system or the potential equation, or from nonclassical solutions generated by applying the Nonclassical Method $(\tau \equiv 1)$ to the given scalar PDE.
\end{abstract}

\section{Introduction}

Suppose a given scalar partial differential equation (PDE) of second order

$$
F\left(x, t, u, u_{x}, u_{t}, u_{x x}, u_{x t}, u_{t t}\right)=0
$$

where the subscripts denote the partial derivatives of $u$, can be written as a conservation law

$$
F\left(x, t, u, u_{x}, u_{t}, u_{x x}, u_{x t}, u_{t t}\right)=\frac{D}{D x} f\left(x, t, u, u_{x}, u_{t}\right)-\frac{D}{D t} g\left(x, t, u, u_{x}, u_{t}\right)=0
$$

for some functions $f$ and $g$ of the indicated arguments. Here, $\frac{D}{D x}$ and $\frac{D}{D t}$ are total derivative operators defined by

$$
\begin{aligned}
\frac{D}{D x} & =\frac{\partial}{\partial x}+u_{x} \frac{\partial}{\partial u}+u_{x x} \frac{\partial}{\partial u_{x}}+u_{x t} \frac{\partial}{\partial u_{t}}+\cdots, \\
\frac{D}{D t} & =\frac{\partial}{\partial t}+u_{t} \frac{\partial}{\partial u}+u_{x t} \frac{\partial}{\partial u_{x}}+u_{t t} \frac{\partial}{\partial u_{t}}+\cdots
\end{aligned}
$$


Through the conservation law (1.2) one can introduce an auxiliary potential variable $v$ and form an auxiliary potential system

$$
\begin{aligned}
& v_{t}=f\left(x, t, u, u_{x}, u_{t}\right), \\
& v_{x}=g\left(x, t, u, u_{x}, u_{t}\right) .
\end{aligned}
$$

For many physical equations one can eliminate $u$ from the potential system $(1.4 \mathrm{a}, \mathrm{b})$ and form an auxiliary potential equation

$$
G\left(x, t, v, v_{x}, v_{t}, v_{x x}, v_{x t}, v_{t t}\right)=0
$$

for some function $G$ of the indicated arguments.

In principle, one has a related potential equation (1.5) if any of the following three conditions holds:

(1) $\left(\frac{\partial f}{\partial u_{x}}\right)^{2}+\left(\frac{\partial f}{\partial u_{t}}\right)^{2}=0$;

(2) $\left(\frac{\partial g}{\partial u_{x}}\right)^{2}+\left(\frac{\partial g}{\partial u_{t}}\right)^{2}=0$;

(3) $\frac{\partial f}{\partial u}=\frac{\partial g}{\partial u}=0$.

Proposition 1.1 The given scalar PDE (1.2) and the potential system (1.4a,b) are equivalent. If the potential equation (1.5) exists, then the systems of PDEs (1.2), (1.4) and (1.5) are equivalent.

Proof (Ia) If $u=\theta(x, t)$ solves (1.2), then from the integrability condition associated with $(1.4 \mathrm{a}, \mathrm{b}), v_{x t}=v_{t x}$, it follows that there exists a corresponding solution $(u, v)=(\theta(x, t), \phi(x, t))$ of system $(1.4 \mathrm{a}, \mathrm{b})$. Moreover $\phi(x, t)$ is unique to within an arbitrary constant.

(Ib) If $(u, v)=(\theta(x, t), \phi(x, t))$ solves $(1.4 \mathrm{a}, \mathrm{b})$, then $u=\theta(x, t)$ solves (1.2).

Now assume that the potential equation (1.5) exists.

(IIa) If $(u, v)=(\theta(x, t), \phi(x, t))$ solves $(1.4 \mathrm{a}, \mathrm{b})$, then $v=\phi(x, t)$ solves $(1.5)$.

(IIb) If $v=\phi(x, t)$ solves (1.5), then from the definition of $(1.4 \mathrm{a}, \mathrm{b})$, it follows that there exists some $u=\theta(x, t)$ such that $(u, v)=(\theta(x, t), \phi(x, t))$ solves $(1.4 \mathrm{a}, \mathrm{b})$.

The statements Iab and IIab lead to:

(IIIa) If $u=\theta(x, t)$ solves (1.2), then to within an arbitrary constant there corresponds a solution $v=\phi(x, t)$ of (1.5), from the integrability condition associated with $(1.4 \mathrm{a}, \mathrm{b})$.

(IIIb) If $v=\phi(x, t)$ solves (1.5), then through IIb there exists $u=\theta(x, t)$ solving (1.2).

In the important special case where $g\left(x, t, u, u_{x}, u_{t}\right)=u$, i.e. PDE (1.2) is of the form $u_{t}=\frac{D}{D x} f\left(x, t, u, u_{x}, u_{t}\right)$, one has $\theta(x, t)=\phi_{x}(x, t)$ in IIb and IIIb. 


\subsection{The classical method: invariant solutions arising from admitted point symmetries}

A point symmetry

$$
X=\xi(x, t, u) \frac{\partial}{\partial x}+\tau(x, t, u) \frac{\partial}{\partial t}+\eta(x, t, u) \frac{\partial}{\partial u}
$$

is admitted by (1.2) if and only if

$$
\left.\left[X^{(2)}(F)\right]\right|_{F=0}=0
$$

where the second extension of $X$ is given by

$$
X^{(2)}=X+\eta^{x} \frac{\partial}{\partial u_{x}}+\eta^{t} \frac{\partial}{\partial u_{t}}+\eta^{x t} \frac{\partial}{\partial u_{x t}}+\eta^{t t} \frac{\partial}{\partial u_{t t}}+\eta^{x x} \frac{\partial}{\partial u_{x x}}
$$

with

$$
\begin{array}{r}
\eta^{x}=\frac{D \eta}{D x}-\frac{D \xi}{D x} u_{x}-\frac{D \tau}{D x} u_{t}, \quad \eta^{t}=\frac{D \eta}{D t}-\frac{D \xi}{D t} u_{x}-\frac{D \tau}{D t} u_{t}, \\
\eta^{x t}=\frac{D \eta^{t}}{D x}-\frac{D \xi}{D x} u_{x t}-\frac{D \tau}{D x} u_{t t}=\frac{D \eta^{x}}{D t}-\frac{D \xi}{D t} u_{x x}-\frac{D \tau}{D t} u_{x t}, \\
\eta^{t t}=\frac{D \eta^{t}}{D t}-\frac{D \xi}{D t} u_{x t}-\frac{D \tau}{D t} u_{t t}, \quad \eta^{x x}=\frac{D \eta^{x}}{D x}-\frac{D \xi}{D x} u_{x x}-\frac{D \tau}{D x} u_{x t} .
\end{array}
$$

Similarly, a point symmetry

$$
X=\xi(x, t, u, v) \frac{\partial}{\partial x}+\tau(x, t, u, v) \frac{\partial}{\partial t}+\eta(x, t, u, v) \frac{\partial}{\partial u}+\zeta(x, t, u, v) \frac{\partial}{\partial v}
$$

is admitted by (1.4) if and only if for the corresponding first extension $X^{(1)}$ of (1.10), we have

a point symmetry

$$
\begin{aligned}
& {\left.\left[X^{(1)}\left(v_{t}-f\right)\right]\right|_{\left(v_{t}, v_{x}\right)=(f, g)}=0,} \\
& {\left.\left[X^{(1)}\left(v_{x}-g\right)\right]\right|_{\left(v_{t}, v_{x}\right)=(f, g)}=0 ;}
\end{aligned}
$$

$$
X=\xi(x, t, v) \frac{\partial}{\partial x}+\tau(x, t, v) \frac{\partial}{\partial t}+\zeta(x, t, v) \frac{\partial}{\partial v}
$$

is admitted by (1.5) if and only if for the corresponding second extension $X^{(2)}$ of (1.12), we have

$$
\left.\left[X^{(2)}(G)\right]\right|_{G=0}=0 .
$$

For details, see Bluman \& Cole [6], Ovsiannikov [17], Olver [16], Bluman \& Kumei [7] and Bluman \& Anco [4].

Note that a point symmetry of the form (1.10) yields a nonlocal symmetry (potential symmetry) of the given PDE (1.2) if $\xi_{v}^{2}+\tau_{v}^{2}+\eta_{v}^{2} \neq 0$; a point symmetry of the form (1.12) yields a nonlocal symmetry (potential symmetry) of (1.2) if $\xi_{v}^{2}+\tau_{v}^{2} \neq 0$ [7].

A point symmetry $X$ admitted by a system of PDEs maps solutions into other solutions of the same system. A solution which maps into itself is called an invariant solution. 
An invariant solution $u=\theta(x, t)$ of PDE (1.2) satisfies the invariant surface condition

$$
\left.[X(u-\theta(x, t))]\right|_{u=\theta(x, t)}=0,
$$

i.e.

$$
\xi(x, t, u) u_{x}+\tau(x, t, u) u_{t}=\eta(x, t, u)
$$

as well as PDE (1.2).

The general solution of the characteristic equations corresponding to the first order scalar PDE (1.15) can be represented in the form

$$
\begin{gathered}
z(x, t, u)=\mathrm{const}=c_{1}(\text { similarity variable }) \\
W(x, t, u)=\mathrm{const}=c_{2}=w(z)
\end{gathered}
$$

yielding an ansatz

$$
u=\Phi(x, t, w(z(x, t, u)))
$$

for solutions of (1.2), after in principle solving (1.16b) in terms of $u$. For a given point symmetry $X$ (1.6) of PDE (1.2), the dependence of $\Phi$ on $x, t$ and $w(z)$ is explicit in (1.17); $w(z)$ is an arbitrary function of the similarity variable $z$. The substitution of (1.17) into (1.2) leads to a reduced ODE of order at most two with independent variable $z$ and dependent variable $w$. Note that $z_{u}=0$ if and only if $\tau=0$ or $\frac{\partial}{\partial u}\left(\frac{\xi}{\tau}\right)=0$.

In the case of the potential system $(1.4 \mathrm{a}, \mathrm{b})$, an admitted point symmetry $X$ given by $(1.10)$ yields invariant solutions $(u, v)=(\theta(x, t), \phi(x, t))$ satisfying the invariant surface conditions

$$
\begin{aligned}
& {\left.[X(u-\theta(x, t))]\right|_{(u, v)=(\theta(x, t), \phi(x, t))}=0,} \\
& {\left.[X(v-\phi(x, t))]\right|_{(u, v)=(\theta(x, t), \phi(x, t))}=0,}
\end{aligned}
$$

i.e.

$$
\begin{aligned}
& \xi(x, t, u, v) u_{x}+\tau(x, t, u, v) u_{t}=\eta(x, t, u, v), \\
& \xi(x, t, u, v) v_{x}+\tau(x, t, u, v) v_{t}=\zeta(x, t, u, v)
\end{aligned}
$$

as well as satisfying system $(1.4 \mathrm{a}, \mathrm{b})$.

The general solution of the characteristic equations corresponding to (1.19) can be represented in the form

$$
\begin{gathered}
z(x, t, u, v)=\mathrm{const}=c_{1}(\text { similarity variable }), \\
W_{1}(x, t, u, v)=\mathrm{const}=c_{2}=w_{1}(z), \\
W_{2}(x, t, u, v)=\mathrm{const}=c_{3}=w_{2}(z),
\end{gathered}
$$

yielding the ansatz

$$
\begin{aligned}
& u=\Phi\left(x, t, w_{1}(z(x, t, u, v)), w_{2}(z(x, t, u, v))\right), \\
& v=\Psi\left(x, t, w_{1}(z(x, t, u, v)), w_{2}(z(x, t, u, v))\right),
\end{aligned}
$$


for solutions of the potential system $(1.4 \mathrm{a}, \mathrm{b})$, after in principle solving $(1.20 \mathrm{~b}, \mathrm{c})$ in terms of $u$ and $v$. For a given point symmetry $X(1.10)$ of potential system $(1.4 \mathrm{a}, \mathrm{b})$, the dependence of $\Phi$ and $\Psi$ on $x, t, w_{1}(z)$ and $w_{2}(z)$ is explicit in $(1.21 \mathrm{a}, \mathrm{b}) ; w_{1}(z)$ and $w_{2}(z)$ are both arbitrary functions of the similarity variable $z$. The substitution of $(1.21 \mathrm{a}, \mathrm{b})$ into $(1.4 \mathrm{a}, \mathrm{b})$ leads to a reduced system of ODEs with independent variable $z$ and dependent variables $w_{1}(z)$ and $w_{2}(z)$. [Note that $z_{u}=z_{v}=0$ if and only if $\tau=0$ or $\frac{\partial}{\partial u}\left(\frac{\xi}{\tau}\right)=\frac{\partial}{\partial v}\left(\frac{\xi}{\tau}\right)=0$.] In particular, we end up with a solution of the form $u=\Phi\left(x, t, w_{1}(z), w_{2}(z)\right)$ of PDE (1.2) with $w_{i}(z)=w_{i}(z(x, t, u, v)), i=1,2$.

In the case of the potential equation (1.5), similar to the situation for the given scalar PDE (1.2), an admitted point symmetry $X$ given by (1.12) yields invariant solutions $v=\phi(x, t)$ of the potential equation (1.5) that satisfy the invariant surface condition

$$
\left.[X(v-\phi(x, t))]\right|_{v=\phi(x, t)}=0
$$

i.e.

$$
\xi(x, t, v) v_{x}+\tau(x, t, v) v_{t}=\zeta(x, t, v)
$$

as well as satisfying (1.5).

The general solution of the characteristic equations corresponding to (1.23) can be represented by

$$
\begin{gathered}
z(x, t, v)=\mathrm{const}=c_{1}(\text { similarity variable }), \\
W(x, t, v)=\mathrm{const}=c_{2}=w_{1}(z),
\end{gathered}
$$

yielding an ansatz

$$
v=\Psi\left(x, t, w_{1}(z(x, t, v))\right)
$$

for solutions to (1.5), after in principle solving (1.24b) in terms of $v$. For a given point symmetry $X$ (1.12) of potential equation (1.5), the dependence of $\Psi$ on $x, t$ and $w_{1}(z)$ is explicit in $(1.25) ; w_{1}(z)$ is an arbitrary function of the similarity variable $z$. The substitution of (1.25) into (1.5) leads to a reduced ODE of order at most two with independent variable $z$ and dependent variable $w_{1}$. Note that $z_{v}=0$ if and only if $\tau=0$ or $\frac{\partial}{\partial v}\left(\frac{\xi}{\tau}\right)=0$.

In the important special case where $g\left(x, t, u, u_{x}, u_{t}\right)=u$, i.e. PDE (1.2) is of the form $u_{t}=\frac{D}{D x} f\left(x, t, u, u_{x}, u_{t}\right)$, the ansatz (1.25) yields a solution of PDE (1.2) of the form

$$
u=v_{x}=\frac{F_{1}\left(x, t, w_{1}(z)\right)+w_{2}(z) F_{2}\left(x, t, w_{1}(z)\right)}{1+w_{2}(z) F_{3}\left(x, t, w_{1}(z)\right)}
$$

in terms of explicit functions

$$
F_{1}=\Psi_{x}, \quad F_{2}=z_{x} \Psi_{w_{1}}, \quad F_{3}=-z_{v} \Psi_{w_{1}}, \quad w_{2}(z)=\frac{d w_{1}(z)}{d z} .
$$

Note that $F_{3}=0$ if $z_{v}=0 ; F_{2}=0$ if $z_{x}=0$. 


\subsection{The nonclassical method: solutions arising from admitted nonclassical symmetries}

The algorithmic Nonclassical Method, introduced in Bluman \& Cole [5], generalizes and includes the Classical Method for obtaining solutions of PDEs. Here we seek all $(\xi(x, t, u), \tau(x, t, u), \eta(x, t, u))$ so that (1.6) is a symmetry (nonclassical symmetry) that leaves invariant the augmented system consisting of (1.2), (1.15) and differential consequences of (1.15). From the discussion of the Classical Method in $\S 1.1$, it follows that the Nonclassical Method yields all solutions of PDE (1.2) of the form $u=\Phi(x, t, w(z(x, t, u)))$ where $w(z)$ satisfies a reduced ODE.

From the nature of the invariant surface condition (1.15), without loss of generality, two cases arise: $\tau \equiv 1 ; \tau \equiv 0, \xi \equiv 1$. Equation (1.15), and its differential consequences, introduce additional relationships between the derivatives of $u$ beyond those used to find the point symmetries of PDE (1.2). For any choice of $(\xi(x, t, u), \tau(x, t, u), \eta(x, t, u))$, (1.6) leaves invariant (1.15) [14]. Consequently, the solutions of PDE (1.2), arising from the nonclassical symmetries of the augmented system, include all invariant solutions of (1.2) arising from the point symmetries of (1.2). One can show that the compatibility of the completely augmented system consisting of the given PDE (1.2), the invariant surface condition (1.15), and the differential consequences of both (1.2) and (1.15), leads to the determining equations of the Nonclassical Method (when applied to the completely augmented system) for $(\xi(x, t, u), \tau(x, t, u), \eta(x, t, u))$.

Definition 1.2 A solution of PDE (1.2) is called a nonclassical solution of (1.2) if it is obtained by the Nonclassical Method and is not an invariant solution arising from an admitted point symmetry of (1.2).

\subsection{Extensions of the nonclassical method: solutions arising from admitted nonclassical potential symmetries}

We now introduce two algorithms which extend the Nonclassical Method to a potential system $(1.4 \mathrm{a}, \mathrm{b})$ or a potential equation $(1.5)$.

\subsubsection{Algorithm I (Potential system approach)}

Here we seek all $(\xi(x, t, u, v), \tau(x, t, u, v), \eta(x, t, u, v), \zeta(x, t, u, v))$ so that $(1.10)$ is a symmetry (nonclassical potential symmetry of the given PDE (1.2)) that leaves invariant the augmented system consisting of $(1.4 \mathrm{a}, \mathrm{b}),(1.19)$ and differential consequences of (1.19). From the discussion of the Classical Method in $\S 1.1$, it follows that we obtain solutions of system $(1.4 \mathrm{a}, \mathrm{b})$ of the form $(u, v)=\left(\Phi\left(x, t, w_{1}(z(x, t, u, v)), w_{2}(z(x, t, u, v))\right)\right.$, $\left.\Psi\left(x, t, w_{1}(z(x, t, u, v)), w_{2}(z(x, t, u, v))\right)\right)$ where $w_{1}(z)$ and $w_{2}(z)$ satisfy a reduced system of ODEs. (Note that if $\xi_{v}^{2}+\tau_{v}^{2}+\eta_{v}^{2} \equiv 0$, then no new solutions of PDE (1.2) are found, i.e. all such solutions of (1.2) are obtained from the Nonclassical Method applied to PDE (1.2).)

From the nature of the invariant surface conditions (1.19), without loss of generality, two cases arise: $\tau \equiv 1 ; \tau \equiv 0, \xi \equiv 1$. System (1.19), and its differential consequences, introduce additional relationships between the derivatives of $u$ and $v$ beyond those used to find the point symmetries of potential system $(1.4 \mathrm{a}, \mathrm{b})$. For any choice of 
$(\xi(x, t, u, v), \tau(x, t, u, v), \eta(x, t, u, v), \zeta(x, t, u, v)),(1.10)$ leaves invariant (1.19). Consequently, the solutions of potential system $(1.4 \mathrm{a}, \mathrm{b})$, arising from the nonclassical symmetries of the augmented system, include all invariant solutions of $(1.4 \mathrm{a}, \mathrm{b})$ that arise from the point symmetries of potential system $(1.4 \mathrm{a}, \mathrm{b})$. One can show that the compatibility of the completely augmented system consisting of the potential system $(1.4 \mathrm{a}, \mathrm{b})$, the invariant surface conditions (1.19), and the differential consequences of both (1.4a,b) and (1.19), leads to the determining equations of the Nonclassical Method (when applied to the completely augmented system) for $(\xi(x, t, u, v), \tau(x, t, u, v), \eta(x, t, u, v), \zeta(x, t, u, v))$.

\subsubsection{Algorithm II (Potential equation approach)}

Here we seek all $(\xi(x, t, v), \tau(x, t, v), \zeta(x, t, v))$ so that (1.12) is a symmetry (nonclassical potential symmetry of the given PDE (1.2)) that leaves invariant the augmented system consisting of the potential equation (1.5), the invariant surface condition (1.23), and differential consequences of (1.23). From the discussion in $\S 1.1$, it follows that we obtain solutions of the given scalar PDE (1.2) related to all solutions of the potential equation (1.5) of the form $v=\Psi\left(x, t, w_{1}(z(x, t, v))\right)$ where $w_{1}(z)$ satisfies a reduced ODE. (In the important special case where $g\left(x, t, u, u_{x}, u_{t}\right)=u$, i.e. PDE (1.2) is of the form $u_{t}=\frac{D}{D x} f\left(x, t, u, u_{x}, u_{t}\right)$, the corresponding solutions of $u_{t}=\frac{D f}{D x}$ are given by (1.26) and (1.27). Even if $\xi_{v}^{2}+\tau_{v}^{2} \equiv 0$, i.e. $z_{v}=0$, we could obtain solutions of PDE (1.2) which are neither invariant solutions of admitted point symmetries of the potential equation (1.5) nor nonclassical solutions of $u_{t}=\frac{D f}{D x}$.)

From the nature of the invariant surface condition (1.23), without loss of generality, two cases arise: $\tau \equiv 1 ; \tau \equiv 0, \xi \equiv 1$. Equation (1.23), and its differential consequences, introduce additional relationships between the derivatives of $v$ beyond those used to find the point symmetries of the potential equation (1.5). For any choice of $(\xi(x, t, v), \tau(x, t, v), \zeta(x, t, v))$, (1.12) leaves invariant (1.23). Consequently, the solutions of (1.5), arising from the nonclassical symmetries of the augmented system, include all invariant solutions of potential equation (1.5) arising from the point symmetries of potential equation (1.5). One can show that the compatibility of the completely augmented system consisting of the potential equation (1.5), the invariant surface condition (1.23), and the differential consequences of both (1.5) and (1.23), leads to the determining equations of the Nonclassical Method (when applied to the completely augmented system) for $(\xi(x, t, v), \tau(x, t, v), \zeta(x, t, v))$.

Definition 1.3 A solution of PDE (1.2) obtained from a nonclassical symmetry with $\tau \equiv 1$ through either Algorithm I or Algorithm II is called a nonclassical potential solution of (1.2) if it is neither a nonclassical solution of (1.2) arising from a nonclassical symmetry with $\tau \equiv 1$ nor a solution obtained from an invariant solution of an admitted point symmetry of the given PDE (1.2), the potential system $(1.4 \mathrm{a}, \mathrm{b})$ or the potential equation (1.5).

Nonclassical potential symmetries arising from the Potential System Approach (Algorithm I) were first discussed in Bluman \& Shtelen [9] and Saccomandi [18], but neither of these papers exhibited nonclassical potential solutions for specific PDEs. Nonclassical potential symmetries arising from the Potential Equation Approach 
(Algorithm II) were first discussed in Gandarias \& Bruzón [13], where no nonclassical potential solutions were found for a family of Cahn-Hilliard equations. In Clarkson \& Priestley [11], using Algorithm I, no nonclassical potential solutions were found for one of the infinite number of potential systems that arise for a shallow water wave equation written in a potential form. Several papers discuss algorithmic procedures for obtaining nonclassical symmetries through symbolic computation (see, for example, Clarkson \& Mansfield [10] and Mansfield et al. [15]).

\subsection{A prototypical example: the nonlinear heat conduction equation}

For the rest of this paper, as a prototypical example, we consider the nonlinear heat conduction equation

$$
u_{t}=\left(K(u) u_{x}\right)_{x}, \quad K^{\prime}(u) \neq 0,
$$

which is already in conservation law form. Correspondingly, we have the potential system

$$
\begin{aligned}
& v_{x}=u, \\
& v_{t}=K(u) u_{x}
\end{aligned}
$$

and the potential equation

$$
v_{t}=K\left(v_{x}\right) v_{x x} .
$$

In $\S 2$, for arbitrary $K(u)$, we set up the determining equations for nonclassical symmetries of the potential equation (1.30), the potential system (1.29), and the given scalar $\operatorname{PDE}$ (1.28), respectively, for $\tau \equiv 1$ and for $\tau \equiv 0, \xi \equiv 1$. When $\tau \equiv 1$, we show that for the potential equation (1.30) to admit nonclassical potential symmetries it is necessary that $K(u)$ be a restricted function depending on at most 11 parameters, whereas the potential system (1.29) admits nonclassical potential symmetries for arbitrary $K(u)$. The determining equations for nonclassical symmetries of the scalar PDE (1.28) appear in Arrigo \& Hill [2] and those for the potential system (1.29) appear in Bluman \& Shtelen [9].

In $\S 3$ and $\S 4$, we consider the case where $K(u)=\frac{1}{u^{2}+u}$ for $\tau \equiv 1$. In $\S 3.1$, we show that the scalar PDE (1.28) only admits nonclassical symmetries which are derivable from its admitted point symmetries. For the corresponding potential equation (1.30) and potential system (1.29), in $\S 3.2$ we list the point symmetries found in Bluman \& Kumei [7], Bluman et al. [8] and Akhatov et al. [1], and in $\S 3.3$ we derive special solution classes of nonclassical symmetries.

In $\S 4$, we obtain solutions of the potential equation (1.30) resulting from a particular subclass of the nonclassical symmetries found in $\S 3.2$. We consider one of these solutions and compare it with all invariant solutions arising from admitted point symmetries of the potential equation (1.30) and the potential system (1.29). Through the comparisons we show that this considered solution yields a nonclassical solution of the potential equation (1.30) and a nonclassical potential solution of the scalar PDE (1.28) for $K(u)=\frac{1}{u^{2}+u}$.

In $\S 5$, we summarize the new results in this paper. We show how our work can be extended to wide classes of higher order PDEs. We also list some important remarks, including comments on related work in Gandarias [12]. 
2 Determining equations for nonclassical symmetries of the nonlinear heat conduction equation for arbitrary $K(u)$

2.1 Determining equations for nonclassical symmetries of the potential equation (1.30) $\tau \equiv 1$

The Nonclassical Method applied to the potential equation (1.30) yields the determining equation for nonclassical symmetries:

$$
\begin{aligned}
& {\left[-\xi \xi_{v} v_{x}^{3}+\left(\xi_{v} \zeta-\xi \xi_{x}+\xi \zeta_{v}\right) v_{x}^{2}+\left(\xi_{x} \zeta-\zeta \zeta_{v}+\xi \zeta_{x}\right) v_{x}-\zeta \zeta_{x}\right] K^{\prime}\left(v_{x}\right)} \\
& \quad+\left[-2 \xi \xi_{v} v_{x}^{2}+\left(2 \xi_{v} \zeta-2 \xi \xi_{x}-\xi_{t}\right) v_{x}+2 \zeta \xi_{x}+\zeta_{t}\right] K\left(v_{x}\right) \\
& \quad+\left[\xi_{v v} v_{x}^{3}+\left(2 \xi_{x v}-\zeta_{v v}\right) v_{x}^{2}+\left(\xi_{x x}-2 \zeta_{x v}\right) v_{x}-\zeta_{x x}\right] K^{2}\left(v_{x}\right)=0 .
\end{aligned}
$$

Since the determining equation (2.1) must hold for all values of $x, t, v$ and $v_{x}$, it follows that $K(u)=K\left(v_{x}\right)$ must satisfy a first order Bernoulli equation (with variable coefficients) of the form

$$
\begin{aligned}
& \left(A_{1} v_{x}^{3}+A_{2} v_{x}^{2}+A_{3} v_{x}+A_{4}\right) K^{\prime}\left(v_{x}\right)+\left(B_{1} v_{x}^{2}+B_{2} v_{x}+B_{3}\right) K\left(v_{x}\right) \\
& \quad+\left(C_{1} v_{x}^{3}+C_{2} v_{x}^{2}+C_{3} v_{x}+C_{4}\right) K^{2}\left(v_{x}\right)=0
\end{aligned}
$$

for some constants $A_{i}, B_{j}, C_{k}$ to be determined. Consequently, $K(u)$ depends at most on 11 parameters.

With the aid of symbolic computation, we eliminate $K^{\prime}\left(v_{x}\right)$ through multiplying (2.1) by $\left(A_{1} v_{x}^{3}+A_{2} v_{x}^{2}+A_{3} v_{x}+A_{4}\right)$ and (2.2) by $\left(-\xi \xi_{v} v_{x}^{3}+\left(\xi_{v} \zeta-\xi \xi_{x}+\xi \zeta_{v}\right) v_{x}^{2}+\left(\xi_{x} \zeta-\zeta \zeta_{v}+\right.\right.$ $\left.\left.\xi \zeta_{x}\right) v_{x}-\zeta \zeta_{x}\right)$, respectively, to obtain

$$
\begin{aligned}
\{ & \left(A_{1} \xi_{v v}+C_{1} \xi \xi_{v}\right) v_{x}^{6}+\left[A_{1}\left(2 \xi_{x v}-\zeta_{v v}\right)+A_{2} \xi_{v v}-C_{1}\left(\xi_{v} \zeta-\xi \xi_{x}+\xi \zeta_{v}\right)+C_{2} \xi \xi_{v}\right] v_{x}^{5} \\
& +\left[A_{1}\left(\xi_{x x}-2 \zeta_{x v}\right)+A_{2}\left(2 \xi_{x v}-\zeta_{v v}\right)+A_{3} \xi_{v v}-C_{1}\left(\xi_{x} \zeta-\zeta \zeta_{v}+\xi \zeta_{x}\right)-C_{2}\left(\xi_{v} \zeta-\xi \xi_{x}+\xi \zeta_{v}\right)\right. \\
& \left.+C_{3} \xi \xi_{v}\right] v_{x}^{4}+\left[-A_{1} \zeta_{x x}+A_{2}\left(\xi_{x x}-2 \zeta_{x v}\right)+A_{3}\left(2 \xi_{x v}-\zeta_{v v}\right)+A_{4} \xi_{v v}+C_{1} \zeta \zeta_{x}\right. \\
& \left.-C_{2}\left(\xi_{x} \zeta-\zeta \zeta_{v}+\xi \zeta_{x}\right)-C_{3}\left(\xi_{v} \zeta-\xi \xi_{x}+\xi \zeta_{v}\right)+C_{4} \xi \xi_{v}\right] v_{x}^{3}+\left[-A_{2} \zeta_{x x}+A_{3}\left(\xi_{x x}-2 \zeta_{x v}\right)\right. \\
& \left.+A_{4}\left(2 \xi_{x v}-\zeta_{v v}\right)+C_{2} \zeta \zeta_{x}-C_{3}\left(\xi_{x} \zeta-\zeta \zeta_{v}+\xi \zeta_{x}\right)-C_{4}\left(\xi_{v} \zeta-\xi \xi_{x}+\xi \zeta_{v}\right)\right] v_{x}^{2} \\
& \left.+\left[-A_{3} \zeta_{x x}+A_{4}\left(\xi_{x x}-2 \zeta_{x v}\right)+C_{3} \zeta \zeta_{x}-C_{4}\left(\xi_{x} \zeta-\zeta \zeta_{v}+\xi \zeta_{x}\right)\right] v_{x}+\left(C_{4} \zeta \zeta_{x}-A_{4} \zeta_{x x}\right)\right\} K\left(v_{x}\right) \\
& +\left\{\left(B_{1}-2 A_{1}\right) \xi \xi_{v} v_{x}^{5}+\left[A_{1}\left(2 \xi_{v} \zeta-2 \xi \xi_{x}-\xi_{t}\right)-2 A_{2} \xi \xi_{v}-B_{1}\left(\xi_{v} \zeta-\xi \xi_{x}+\xi \zeta_{v}\right)+B_{2} \xi \xi_{v}\right] v_{x}^{4}\right. \\
& +\left[A_{1}\left(2 \xi_{x} \zeta+\zeta_{t}\right)+A_{2}\left(2 \xi_{v} \zeta-2 \xi \xi_{x}-\xi_{t}\right)-2 A_{3} \xi \xi_{v}-B_{1}\left(\xi_{x} \zeta-\zeta \zeta_{v}+\xi \zeta_{x}\right)\right. \\
& \left.-B_{2}\left(\xi_{v} \zeta-\xi \xi_{x}+\xi \zeta_{v}\right)+B_{3} \xi \xi_{v}\right] v_{x}^{3}+\left[A_{2}\left(2 \xi_{x} \zeta+\zeta_{t}\right)+A_{3}\left(2 \xi_{v} \zeta-2 \xi \xi_{x}-\xi_{t}\right)\right. \\
& \left.-2 A_{4} \xi \xi_{v}+B_{1} \zeta \zeta_{x}-B_{2}\left(\xi_{x} \zeta-\zeta \zeta_{v}+\xi \zeta_{x}\right)-B_{3}\left(\xi_{v} \zeta-\xi \xi_{x}+\xi \zeta_{v}\right)\right] v_{x}^{2} \\
& +\left[A_{3}\left(2 \xi_{x} \zeta+\zeta_{t}\right)+A_{4}\left(2 \xi_{v} \zeta-2 \xi \xi_{x}-\xi_{t}\right)+B_{2} \zeta \zeta_{x}-B_{3}\left(\xi_{x} \zeta-\zeta \zeta_{v}+\xi \zeta_{x}\right)\right] v_{x} \\
& \left.+\left[A_{4}\left(2 \xi_{x} \zeta+\zeta_{t}\right)+B_{3} \zeta \zeta_{x}\right]\right\}=0 .
\end{aligned}
$$

Equation (2.3) is an expression of the form

$$
\begin{gathered}
\left(\alpha_{1} v_{x}^{6}+\alpha_{2} v_{x}^{5}+\alpha_{3} v_{x}^{4}+\alpha_{4} v_{x}^{3}+\alpha_{5} v_{x}^{2}+\alpha_{6} v_{x}+\alpha_{7}\right) K\left(v_{x}\right) \\
+\beta_{1} v_{x}^{5}+\beta_{2} v_{x}^{4}+\beta_{3} v_{x}^{3}+\beta_{4} v_{x}^{2}+\beta_{5} v_{x}+\beta_{6}=0
\end{gathered}
$$


where each $\alpha_{i}$ and $\beta_{j}$ is the coefficient of the corresponding term of (2.3). From (2.4), two possible cases arise according to whether or not $\sum_{i=1}^{7} \alpha_{i}^{2} \equiv 0$.

Case I. $\sum_{i=1}^{7} \alpha_{i}^{2} \equiv 0$.

Here it must follow that $\beta_{j} \equiv 0(j=1,2, \ldots, 6)$. Then (2.4) splits into the determining equations

$$
\begin{gathered}
A_{1} \xi_{v v}+C_{1} \xi \xi_{v}=0 \\
A_{1}\left(2 \xi_{x v}-\zeta_{v v}\right)+A_{2} \xi_{v v}-C_{1}\left(\xi_{v} \zeta-\xi \xi_{x}+\xi \zeta_{v}\right)+C_{2} \xi \xi_{v}=0, \\
A_{1}\left(\xi_{x x}-2 \zeta_{x v}\right)+A_{2}\left(2 \xi_{x v}-\zeta_{v v}\right)+A_{3} \xi_{v v}-C_{1}\left(\xi_{x} \zeta-\zeta \zeta_{v}+\xi \zeta_{x}\right) \\
-C_{2}\left(\xi_{v} \zeta-\xi \xi_{x}+\xi \zeta_{v}\right)+C_{3} \xi \xi_{v}=0 \\
-A_{1} \zeta_{x x}+A_{2}\left(\xi_{x x}-2 \zeta_{x v}\right)+A_{3}\left(2 \xi_{x v}-\zeta_{v v}\right)+A_{4} \xi_{v v}+C_{1} \zeta \zeta_{x} \\
-C_{2}\left(\xi_{x} \zeta-\zeta \zeta_{v}+\xi \zeta_{x}\right)-C_{3}\left(\xi_{v} \zeta-\xi \xi_{x}+\xi \zeta_{v}\right)+C_{4} \xi \xi_{v}=0, \\
-A_{2} \zeta_{x x}+A_{3}\left(\xi_{x x}-2 \zeta_{x v}\right)+A_{4}\left(2 \xi_{x v}-\zeta_{v v}\right)+C_{2} \zeta \zeta_{x} \\
-C_{3}\left(\xi_{x} \zeta-\zeta \zeta_{v}+\xi \zeta_{x}\right)-C_{4}\left(\xi_{v} \zeta-\xi \xi_{x}+\xi \zeta_{v}\right)=0 \\
-A_{3} \zeta_{x x}+A_{4}\left(\xi_{x x}-2 \zeta_{x v}\right)+C_{3} \zeta \zeta_{x}-C_{4}\left(\xi_{x} \zeta-\zeta \zeta_{v}+\xi \zeta_{x}\right)=0, \\
-A_{4} \zeta_{x x}+C_{4} \zeta \zeta_{x}=0 \\
\left(B_{1}-2 A_{1}\right) \xi \xi_{v}=0 \\
A_{1}\left(2 \xi_{v} \zeta-2 \xi \xi_{x}-\xi_{t}\right)-2 A_{2} \xi \xi_{v}-B_{1}\left(\xi_{v} \zeta-\xi \xi_{x}+\xi \zeta_{v}\right)+B_{2} \xi \xi_{v}=0, \\
A_{1}\left(2 \xi_{x} \zeta+\zeta_{t}\right)+A_{2}\left(2 \xi_{v} \zeta-2 \xi \xi_{x}-\xi_{t}\right)-2 A_{3} \xi \xi_{v}-B_{1}\left(\xi_{x} \zeta-\zeta \zeta_{v}+\xi \zeta_{x}\right) \\
-B_{2}\left(\xi_{v} \zeta-\xi \xi_{x}+\xi \zeta_{v}\right)+B_{3} \xi \xi_{v}=0 \\
A_{2}\left(2 \xi_{x} \zeta+\zeta_{t}\right)+A_{3}\left(2 \xi_{v} \zeta-2 \xi \xi_{x}-\xi_{t}\right)-2 A_{4} \xi \xi_{v}+B_{1} \zeta \zeta_{x} \\
-B_{2}\left(\xi_{x} \zeta-\zeta \zeta_{v}+\xi \zeta_{x}\right)-B_{3}\left(\xi_{v} \zeta-\xi \xi_{x}+\xi \zeta_{v}\right)=0 \\
A_{3}\left(2 \xi_{x} \zeta+\zeta_{t}\right)+A_{4}\left(2 \xi_{v} \zeta-2 \xi \xi_{x}-\xi_{t}\right)+B_{2} \zeta \zeta_{x}-B_{3}\left(\xi_{x} \zeta-\zeta \zeta_{v}+\xi \zeta_{x}\right)=0 \\
A_{4}\left(2 \xi_{x} \zeta+\zeta_{t}\right)+B_{3} \zeta \zeta_{x}=0
\end{gathered}
$$

Case II. $\sum_{i=1}^{7} \alpha_{i}^{2} \neq 0$.

Here (2.4) can be rewritten in the form

$$
K\left(v_{x}\right)=-\frac{\beta_{1} v_{x}^{5}+\beta_{2} v_{x}^{4}+\beta_{3} v_{x}^{3}+\beta_{4} v_{x}^{2}+\beta_{5} v_{x}+\beta_{6}}{\alpha_{1} v_{x}^{6}+\alpha_{2} v_{x}^{5}+\alpha_{3} v_{x}^{4}+\alpha_{4} v_{x}^{3}+\alpha_{5} v_{x}^{2}+\alpha_{6} v_{x}+\alpha_{7}} .
$$

With the aid of symbolic computation, we substitute (2.6) into (2.2) to obtain a polynomial of degree 13 in $v_{x}$. After equating to zero the coefficients of like powers of $v_{x}$, equation (2.2) splits into an overdetermined nonlinear system of 14 PDEs for the unknowns $\xi(x, t, v)$ and $\zeta(x, t, v)$ and undetermined constants $A_{i}, B_{j}, C_{k}$. In general, most of these 14 PDEs involve many terms. For example, the coefficient of $v_{x}^{2}$ yields the determining equation

$$
\begin{aligned}
& {\left[A_{4}\left(2 \xi_{x} \zeta+\zeta_{t}\right)+B_{3} \zeta \zeta_{x}\right]\left\{C_{2}\left[A_{4}\left(2 \xi_{x} \zeta+\zeta_{t}\right)+B_{3} \zeta \zeta_{x}\right]+2 C_{3}\left[A_{3}\left(2 \xi_{x} \zeta+\zeta_{t}\right)+B_{2} \zeta \zeta_{x}\right.\right.} \\
& \left.\quad+A_{4}\left(2 \xi_{v} \zeta-2 \xi \xi_{x}-\xi_{t}\right)-B_{3}\left(\xi_{x} \zeta-\zeta \zeta_{v}+\xi \zeta_{x}\right)\right]+2 C_{4}\left[A_{2}\left(2 \xi_{x} \zeta+\zeta_{t}\right)-2 A_{4} \xi \xi_{v}\right. \\
& \left.\quad+A_{3}\left(2 \xi_{v} \zeta-2 \xi \xi_{x}-\xi_{t}\right)+B_{1} \zeta \zeta_{x}-B_{2}\left(\xi_{x} \zeta-\zeta \zeta_{v}+\xi \zeta_{x}\right)-B_{3}\left(\xi_{v} \zeta-\xi \xi_{x}+\xi \zeta_{v}\right)\right]
\end{aligned}
$$




$$
\begin{aligned}
& -B_{1}\left(-A_{4} \zeta_{x x}+C_{4} \zeta \zeta_{x}\right)+\left(A_{2}-B_{2}\right)\left[-A_{3} \zeta_{x x}+A_{4}\left(\xi_{x x}-2 \zeta_{x v}\right)+C_{3} \zeta \zeta_{x}\right. \\
& \left.-C_{4}\left(\xi_{x} \zeta-\zeta \zeta_{v}+\xi \zeta_{x}\right)\right]+\left(2 A_{3}-B_{3}\right)\left[-A_{2} \zeta_{x x}+A_{3}\left(\xi_{x x}-2 \zeta_{x v}\right)+A_{4}\left(2 \xi_{x v}-\zeta_{v v}\right)+C_{2} \zeta \zeta_{x}\right. \\
& \left.-C_{3}\left(\xi_{x} \zeta-\zeta \zeta_{v}+\xi \zeta_{x}\right)-C_{4}\left(\xi_{v} \zeta-\xi \xi_{x}+\xi \zeta_{v}\right)\right]+3 A_{4}\left[-A_{1} \zeta_{x x}+A_{2}\left(\xi_{x x}-2 \zeta_{x v}\right)\right. \\
& +A_{3}\left(2 \xi_{x v}-\zeta_{v v}\right)+A_{4} \xi_{v v}+C_{1} \zeta \zeta_{x}-C_{2}\left(\xi_{x} \zeta-\zeta \zeta_{v}+\xi \zeta_{x}\right)-C_{3}\left(\xi_{v} \zeta-\xi \xi_{x}+\xi \zeta_{v}\right) \\
& \left.\left.+C_{4} \xi \xi_{v}\right]\right\}+\left[A_{3}\left(2 \xi_{x} \zeta+\zeta_{t}\right)+A_{4}\left(2 \xi_{v} \zeta-2 \xi \xi_{x}-\xi_{t}\right)+B_{2} \zeta \zeta_{x}\right. \\
& \left.-B_{3}\left(\xi_{x} \zeta-\zeta \zeta_{v}+\xi \zeta_{x}\right)\right]\left\{C _ { 4 } \left[A_{3}\left(2 \xi_{x} \zeta+\zeta_{t}\right)+A_{4}\left(2 \xi_{v} \zeta-2 \xi \xi_{x}-\xi_{t}\right)+B_{2} \zeta \zeta_{x}\right.\right. \\
& \left.-B_{3}\left(\xi_{x} \zeta-\zeta \zeta_{v}+\xi \zeta_{x}\right)\right]-\left(A_{2}+B_{2}\right)\left(-A_{4} \zeta_{x x}+C_{4} \zeta \zeta_{x}\right)-B_{3}\left[-A_{3} \zeta_{x x}+A_{4}\left(\xi_{x x}-2 \eta_{x v}\right)\right. \\
& \left.+C_{3} \zeta \zeta_{x}-C_{4}\left(\xi_{x} \zeta-\zeta \zeta_{v}+\xi \zeta_{x}\right)\right]+A_{4}\left[-A_{2} \zeta_{x x}+A_{3}\left(\xi_{x x}-2 \zeta_{x v}\right)+A_{4}\left(2 \xi_{x v}-\zeta_{v v}\right)+C_{2} \zeta \zeta_{x}\right. \\
& \left.\left.-C_{3}\left(\xi_{x} \zeta-\zeta \zeta_{v}+\xi \zeta_{x}\right)-C_{4}\left(\xi_{v} \zeta-\xi \xi_{x}+\xi \zeta_{v}\right)\right]-\left(A_{2}+B_{2}\right)\left(-A_{4} \zeta_{x x}+C_{4} \zeta \zeta_{x}\right)\right\} \\
& +\left[A_{2}\left(2 \xi_{x} \zeta+\zeta_{t}\right)+A_{3}\left(2 \xi_{v} \zeta-2 \xi \xi_{x}-\xi_{t}\right)-2 A_{4} \xi \xi_{v}+B_{1} \zeta \zeta_{x}-B_{2}\left(\xi_{x} \zeta-\zeta \zeta_{v}+\xi \zeta_{x}\right)\right. \\
& \left.-B_{3}\left(\xi_{v} \zeta-\xi \xi_{x}+\xi \zeta_{v}\right)\right]\left\{-\left(2 A_{3}+B_{3}\right)\left(-A_{4} \zeta_{x x}+C_{4} \zeta \zeta_{x}\right)-A_{4}\left[-A_{3} \zeta_{x x}+A_{4}\left(\xi_{x x}-2 \zeta_{x v}\right)\right.\right. \\
& \left.\left.+C_{3} \zeta \zeta_{x}-C_{4}\left(\xi_{x} \zeta-\zeta \zeta_{v}+\xi \zeta_{x}\right)\right]\right\}-3 A_{4}\left(-A_{4} \zeta_{x x}+C_{4} \zeta \zeta_{x}\right)\left[A_{1}\left(2 \xi_{x} \zeta+\zeta_{t}\right)\right. \\
& -2 A_{3} \xi \xi_{v}+A_{2}\left(2 \xi_{v} \zeta-2 \xi \xi_{x}-\xi_{t}\right)-B_{1}\left(\xi_{x} \zeta-\zeta \zeta_{v}+\xi \zeta_{x}\right) \\
& \left.-B_{2}\left(\xi_{v} \zeta-\xi \xi_{x}+\xi \zeta_{v}\right)+B_{3} \xi \xi_{v}\right]=0 . \\
\tau & 0, \xi \equiv 1
\end{aligned}
$$

Here the invariant surface condition (1.23) becomes $v_{x}=\zeta(x, t, v)$. The corresponding determining equation for nonclassical symmetries is given by

$$
\left(2 \zeta \zeta_{x} \zeta_{v}+\zeta^{2} \zeta_{v}^{2}+\zeta_{x}^{2}\right) K^{\prime}(\zeta)+\left(\zeta^{2} \zeta_{v v}+2 \zeta \zeta_{x v}+\zeta_{x x}\right) K(\zeta)-\zeta_{t}=0 .
$$

In principle, any $K(u)=K(\zeta)$ yields solutions of (2.8). In practice, one must use ansatzes for $\zeta(x, t, v)$ to seek particular solutions.

\subsection{Determining equations for nonclassical symmetries of the potential system (1.29)} $\tau \equiv 1$

The Nonclassical Method applied to the potential system (1.29) yields the system of two determining equations for nonclassical symmetries:

$$
\begin{gathered}
\frac{1}{K(u)}(\zeta-u \xi)\left(\zeta_{u}-u \xi_{u}\right)+u\left(\zeta_{v}-\xi_{x}\right)-\xi_{v} u^{2}-\eta+\zeta_{x}=0 \\
\left(\zeta_{u}-u \xi_{u}\right)\left[\eta-\frac{1}{K(u)} \xi(\zeta-u \xi)\right]+\left(\zeta_{v}-u \xi_{v}\right)(\zeta-u \xi)-\left(K(u) \eta_{v}+\xi_{t}\right) u \\
-K(u) \eta_{x}+\frac{1}{K(u)}(\zeta-u \xi)\left[K(u) \xi_{x}+K(u) \xi_{v} u-K^{\prime}(u) \eta-K(u) \eta_{u}\right] \\
+\frac{1}{K(u)} \xi_{u}(\zeta-u \xi)^{2}+\zeta_{t}=0
\end{gathered}
$$

The determining equations (2.9a,b) first appeared in Saccomandi [18] and are clearly underdetermined since they involve two equations in the three unknowns $\xi(x, t, u, v), \eta(x, t$, $u, v)$ and $\zeta(x, t, u, v)$. Consequently, any $K(u)$ yields, in principle, an infinite number of nonclassical symmetries. We have been unsuccessful in finding a specific solution yielding a nonclassical symmetry that is not derivable from a point symmetry admitted by the 
potential system (1.29). The point symmetries admitted by the potential system (1.29) are given in Bluman \& Kumei [7] and Bluman et al. [8].

$\tau \equiv 0, \xi \equiv 1$

Here it is easy to show that the Nonclassical Method only yields solutions of the potential system (1.29) of the form $u=f(x)$, i.e. invariant solutions obtained from the invariance of (1.29) under translations in $t$.

\subsection{Determining equations for nonclassical symmetries of the given scalar PDE (1.28)}

$\tau \equiv 1$

The Nonclassical Method applied to the given nonlinear heat conduction equation (1.28) yields the following four determining equations for the unknowns $\xi(x, t, u)$ and $\eta(x, t, u)$ :

$$
\begin{gathered}
K^{\prime}(u) \xi_{u}-K(u) \xi_{u u}=0 \\
{\left[K(u) K^{\prime \prime}(u)-K^{\prime 2}(u)\right] \eta+K(u) K^{\prime}(u) \eta_{u}+2 K(u) \xi \xi_{u}+K^{2}(u)\left(\eta_{u u}-2 \xi_{x u}\right)=0} \\
K(u) \xi_{t}-2 K(u) \xi_{u} \eta-K^{\prime}(u) \xi \eta+K^{2}(u)\left(2 \eta_{x u}-\xi_{x x}\right)+2 K(u) \xi \xi_{x}+2 K(u) K^{\prime}(u) \eta_{x}=0 \\
K^{\prime}(u) \eta^{2}-2 K(u) \eta \xi_{x}+K^{2}(u) \eta_{x x}-K(u) \eta_{t}=0
\end{gathered}
$$

$\tau \equiv 0, \xi \equiv 1$

Here the Nonclassical Method yields the determining equation

$$
K(u)\left(\eta_{x x}+2 \eta \eta_{x u}+\eta^{2} \eta_{u u}\right)+K^{\prime \prime}(u) \eta^{3}+K^{\prime}(u)\left(3 \eta \eta_{x}+2 \eta^{2} \eta_{u}\right)-\eta_{t}=0
$$

for the unknown $\eta(x, t, u)$. The systems of determining equations (2.10a-d) and (2.11) first appeared in Bluman \& Shtelen [9]. In principle, any $K(u)$ yields solutions of (2.11). In practise, one must use ansatzes for $\eta(x, t, u)$ to seek particular solutions.

\section{The Case $K(u)=\frac{1}{u^{2}+u}$}

For the rest of this paper, we consider symmetries of (1.28)-(1.30) with the conductivity $K(u)=\frac{1}{u^{2}+u}$.

\subsection{Nonclassical symmetries of the scalar equation (1.28) when $\tau \equiv 1$}

For $K(u)=\frac{1}{u^{2}+u}$, one can show that the only solution of the nonclassical symmetry determining equations $(2.10 \mathrm{a}-\mathrm{d})$ for the nonlinear heat conduction equation (1.28) is given by $\xi=\frac{c_{3} x+c_{2}}{2 c_{3} t+c_{1}}, \zeta=0$. This corresponds to the nonclassical symmetry

$$
Y=\frac{c_{3} x+c_{2}}{2 c_{3} t+c_{1}} \frac{\partial}{\partial x}+\frac{\partial}{\partial t},
$$

where $c_{1}, c_{2}$ and $c_{3}$ are arbitrary constants with $c_{1}^{2}+c_{3}^{2} \neq 0$.

It is easy to see that the nonclassical symmetry (3.1) is derivable from the invariance of PDE (1.28) under the three-parameter Lie group of translations in $x$, translations in $t$ and scalings $x \rightarrow \alpha x, t \rightarrow \alpha^{2}$. Hence the Nonclassical Method applied to the scalar PDE (1.28), when $\tau \equiv 1$, yields no nonclassical solutions of (1.28). 


\subsection{Point symmetries of the potential equation (1.30) and potential system (1.29)}

By applying the classical Lie's algorithm to (1.30), one obtains the point symmetries of (1.30):

$$
\begin{gathered}
X_{1}=\frac{\partial}{\partial v}, \quad X_{2}=\frac{\partial}{\partial x}, \quad X_{3}=\frac{\partial}{\partial t}, \quad X_{4}=x \frac{\partial}{\partial x}+2 t \frac{\partial}{\partial t}+v \frac{\partial}{\partial v}, \\
X_{5}=v \frac{\partial}{\partial x}-t \frac{\partial}{\partial t}-v \frac{\partial}{\partial v}
\end{gathered}
$$

which can be rewritten in the five-parameter form

$$
X=\left(a_{2}+a_{4} x+a_{5} v\right) \frac{\partial}{\partial x}+\left[a_{3}+\left(2 a_{4}-a_{5}\right) t\right] \frac{\partial}{\partial t}+\left[a_{1}+\left(a_{4}-a_{5}\right) v\right] \frac{\partial}{\partial v}
$$

where each $a_{i}(i=1,2, \ldots, 5)$ is an arbitrary constant [7,1].

Lie's algorithm applied to the potential system (1.29) yields the admitted point symmetries ([7], [8])

$$
\begin{gathered}
X_{1}=\frac{\partial}{\partial v}, \quad X_{2}=\frac{\partial}{\partial x}, \quad X_{3}=\frac{\partial}{\partial t}, \quad X_{4}=x \frac{\partial}{\partial x}+2 t \frac{\partial}{\partial t}+v \frac{\partial}{\partial v}, \\
X_{5}=v \frac{\partial}{\partial x}-t \frac{\partial}{\partial t}-v \frac{\partial}{\partial v}-\left(u^{2}+u\right) \frac{\partial}{\partial u} .
\end{gathered}
$$

By comparing the infinitesimal generators (3.2) and (3.4), one sees that the resulting invariant solutions of (1.30) and (1.29), respectively, yield the same solutions of the given nonlinear heat conduction equation (1.28). Moreover, by comparing the infinitesimal generators (3.3) and (3.1), we see that the invariant solutions of the potential equation (1.30) obtained from (3.3) include as a subclass all solutions of the given scalar PDE (1.28) obtained as invariant solutions from its admitted point symmetries equivalent to (3.1).

\subsection{Special classes of nonclassical symmetries of the potential equation (1.30)}

In general, it is difficult to find all solutions $\left(\xi\left(x, t, v, A_{i}, B_{j}, C_{k}\right), \zeta\left(x, t, v, A_{i}, B_{j}, C_{k}\right)\right)$ of the overdetermined nonlinear system of PDEs $(2.5 \mathrm{a}-\mathrm{m})$. Since system $(2.5 \mathrm{a}-\mathrm{m})$ is invariant under translations in $x, t$ and $v$, we seek corresponding invariant solutions of system $(2.5 \mathrm{a}-\mathrm{m})$ of the form $(\xi(\beta v+\gamma x+\lambda t+c), \zeta(\beta v+\gamma x+\lambda t+c))$. Next we consider the following solutions of $(2.5 \mathrm{a}, \mathrm{g})$ :

$$
\begin{aligned}
& \xi=\xi_{1}=b \tanh [b(\beta v+\gamma x+\lambda t)+c], \\
& \xi=\xi_{2}=b \operatorname{coth}[b(\beta v+\gamma x+\lambda t)+c], \\
& \zeta=\zeta_{1}=\alpha b \tanh [b(\beta v+\gamma x+\lambda t)+c], \\
& \zeta=\zeta_{2}=\alpha b \operatorname{coth}[b(\beta v+\gamma x+\lambda t)+c]
\end{aligned}
$$

with the undetermined constants $A_{1}=-\beta, C_{1}=-2 \beta^{2}, A_{4}=-\alpha^{2} \gamma, C_{4}=2 \alpha \gamma^{2}$. The constants $\alpha, b, \beta, \gamma, \lambda, c$ are to be determined. 
The substitution of the solution pairs $\left(\xi_{1}, \zeta_{1}\right),\left(\xi_{2}, \zeta_{1}\right),\left(\xi_{1}, \zeta_{2}\right)$ and $\left(\xi_{2}, \zeta_{2}\right)$, respectively, into equation $(2.5 \mathrm{~m})$ yields the only possible solution pairs $\left(\xi_{1}, \zeta_{1}\right)$ and $\left(\xi_{2}, \zeta_{2}\right)$ with $\lambda=0, B_{3}=2 \alpha \gamma$. Then the remaining ten equations of $(2.5 \mathrm{a}-\mathrm{m})$ yield the solution pairs

$$
\begin{aligned}
& \left(\xi_{1}, \zeta_{1}\right)=(b \tanh [b(\beta v+\gamma x)+c], \alpha b \tanh [b(\beta v+\gamma x)+c]), \\
& \left(\xi_{2}, \zeta_{2}\right)=(b \operatorname{coth}[b(\beta v+\gamma x)+c], \alpha b \operatorname{coth}[b(\beta v+\gamma x)+c])
\end{aligned}
$$

with the undetermined constants $A_{i}, B_{j}, C_{k}$ given by

$$
\begin{gathered}
A_{1}=-\beta, \quad A_{2}=2 \alpha \beta-\gamma, \quad A_{3}=2 \alpha \gamma-\alpha^{2} \beta, \quad A_{4}=-\alpha^{2} \gamma, \\
B_{1}=-2 \beta, \quad B_{2}=2 \alpha \beta-2 \gamma, \quad B_{3}=2 \alpha \gamma, \\
C_{1}=-2 \beta^{2}, \quad C_{2}=2 \alpha \beta^{2}-4 \beta \gamma, \quad C_{3}=-2 \gamma^{2}+4 \alpha \beta \gamma, \quad C_{4}=2 \alpha \gamma^{2} .
\end{gathered}
$$

The substitution of (3.7) or (3.8) with (3.9) into (2.2) yields

$$
\left(v_{x}-\alpha\right) K^{\prime}\left(v_{x}\right)+2 K\left(v_{x}\right)+\left(2 \beta v_{x}+2 \gamma\right) K^{2}\left(v_{x}\right)=0 .
$$

It is easy to see that the general solution of (3.10) is given by

$$
K\left(v_{x}\right)=\frac{1}{A v_{x}^{2}+B v_{x}+C}
$$

where

$$
B=-2 A \alpha-2 \beta, \quad C=A \alpha^{2}-\gamma+\alpha \beta
$$

and $A$ is an arbitrary constant.

If $A=1, \alpha=-2 \gamma, \beta=2 \gamma-\frac{1}{2}$, then we see that the potential equation (1.30) with

$$
K\left(v_{x}\right)=\frac{1}{v_{x}^{2}+v_{x}}
$$

admits two nonclassical symmetries

$$
\begin{gathered}
Y_{1}=b \tanh \left[b\left(2 \gamma-\frac{1}{2}\right) v+b \gamma x+c\right] \frac{\partial}{\partial x}+\frac{\partial}{\partial t}-2 \gamma b \tanh \left[b\left(2 \gamma-\frac{1}{2}\right) v+b \gamma x+c\right] \frac{\partial}{\partial v}, \\
Y_{2}=b \operatorname{coth}\left[b\left(2 \gamma-\frac{1}{2}\right) v+b \gamma x+c\right] \frac{\partial}{\partial x}+\frac{\partial}{\partial t}-2 \gamma b \operatorname{coth}\left[b\left(2 \gamma-\frac{1}{2}\right) v+b \gamma x+c\right] \frac{\partial}{\partial v} .
\end{gathered}
$$

\section{Comparisons of solutions for the case $K(u)=\frac{1}{u^{2}+u}$}

In this section, we obtain solutions of the potential equation (1.30) resulting from the nonclassical symmetries $(3.14 \mathrm{a}, \mathrm{b})$. We consider one of these solutions and compare it with all invariant solutions arising from admitted point symmetries of the potential equation (1.30). Through these comparisons we show that this considered solution yields a nonclassical solution of the potential equation (1.30) and a nonclassical potential solution of the scalar PDE (1.28) for $K(u)=\frac{1}{u^{2}+u}$. 


\subsection{Solutions of the potential equation (1.30) obtained from the nonclassical symmetries $(3.14 a, b)$}

Now consider the nonclassical symmetries $(3.14 \mathrm{a}, \mathrm{b})$ with $b \neq 0, \gamma \neq 0, \frac{1}{2}$. (One can show that if $b=0, \gamma=0$, or $\gamma=\frac{1}{2}$, then the corresponding nonclassical symmetries only yield classical solutions of the potential equation (1.30), i.e. invariant solutions obtained from point symmetries admitted by (1.30).) The characteristic equations corresponding to the invariant surface conditions for the nonclassical symmetries $(3.14 \mathrm{a}, \mathrm{b})$, respectively, are given by

$$
\begin{aligned}
& \frac{d x}{b \tanh \left[b\left(2 \gamma-\frac{1}{2}\right) v+b \gamma x+c\right]}=\frac{d t}{1}=\frac{d v}{-2 \gamma b \tanh \left[b\left(2 \gamma-\frac{1}{2}\right) v+b \gamma x+c\right]} \\
& \frac{d x}{b \operatorname{coth}\left[b\left(2 \gamma-\frac{1}{2}\right) v+b \gamma x+c\right]}=\frac{d t}{1}=\frac{d v}{-2 \gamma b \operatorname{coth}\left[b\left(2 \gamma-\frac{1}{2}\right) v+b \gamma x+c\right]}
\end{aligned}
$$

The resulting similarity variable and similarity forms are given by

$$
\begin{aligned}
\left|\sinh \left[b\left(2 \gamma-\frac{1}{2}\right) v+b \gamma x+c\right]\right| & =e^{b^{2}\left(-4 \gamma^{2}+2 \gamma\right) t} F(Z), & Z=v+2 \gamma x, \\
\cosh \left[b\left(2 \gamma-\frac{1}{2}\right) v+b \gamma x+c\right] & =e^{b^{2}\left(-4 \gamma^{2}+2 \gamma\right) t} F(Z), & Z=v+2 \gamma x .
\end{aligned}
$$

The substitution of (4.2a) or (4.2b) into the potential equation (1.30) with $K\left(v_{x}\right)=\frac{1}{v_{x}^{2}+v_{x}}$ leads to $F(Z)$ satisfying the ODE

$$
F^{\prime \prime}(Z)-\frac{1}{4} b^{2} F(Z)=0
$$

The solution of (4.3) yields two families of solutions of the potential equation (1.30):

$$
\begin{aligned}
& \left|\sinh \left[b\left(2 \gamma-\frac{1}{2}\right) v+b \gamma x+c\right]\right| \\
& =e^{b^{2}\left(-4 \gamma^{2}+2 \gamma\right) t}\left[b_{1} \sinh \left(\frac{1}{2} b v+b \gamma x\right)+b_{2} \cosh \left(\frac{1}{2} b v+b \gamma x\right)\right], \\
& \cosh \left[b\left(2 \gamma-\frac{1}{2}\right) v+b \gamma x+c\right] \\
& =e^{b^{2}\left(-4 \gamma^{2}+2 \gamma\right) t}\left[\delta_{1} \sinh \left(\frac{1}{2} b v+b \gamma x\right)+\delta_{2} \cosh \left(\frac{1}{2} b v+b \gamma x\right)\right],
\end{aligned}
$$

where $b, c, \gamma, b_{1}, b_{2}, \delta_{1}, \delta_{2}$ are arbitrary constants with $b \neq 0, \gamma \neq 0, \frac{1}{2}, b_{2}>\left|b_{1}\right|, \delta_{2}>\left|\delta_{1}\right|$.

\subsection{Classical solutions of the potential equation (1.30)}

We now find the invariant solutions of the potential equation (1.30) resulting from its admitted five-parameter point symmetry (3.3). The characteristic system for the corresponding invariant surface condition is given by

$$
\frac{d x}{a_{2}+a_{4} x+a_{5} v}=\frac{d t}{a_{3}+\left(2 a_{4}-a_{5}\right) t}=\frac{d v}{a_{1}+\left(a_{4}-a_{5}\right) v} .
$$


Table 1. Results table

\begin{tabular}{|c|c|c|c|}
\hline Case & Similarity variable & Similarity form of solution & ODE satisfied by $F(Z)$ \\
\hline $\begin{aligned} a_{4} & \neq 0 \\
\mathrm{I}: & a_{5} \neq a_{4} \\
& a_{5} \neq 2 a_{4}\end{aligned}$ & $\begin{aligned} Z & =\left(v+x-c_{1}\right. \\
& \left.-c_{1}-c_{2}\right)\left(v-c_{1}\right)^{\alpha}\end{aligned}$ & $\begin{array}{l}\left(v-c_{1}\right)^{(1-\alpha)}=\left(t-c_{3}\right) F(Z), \\
c_{1}=\frac{a_{1}}{a_{5}-a_{4}}, \\
c_{2}=-\frac{a_{2}+a_{1} c_{1}}{a_{4}}, \\
c_{3}=\frac{a_{3}}{a_{5}-2 a_{4}}, \alpha=\frac{a_{4}}{a_{5}-a_{4}} \neq 0,1\end{array}$ & $\begin{aligned} F(Z)= & \frac{F^{\prime}(Z)}{(1-\alpha) F(Z)-\alpha Z F^{\prime}(Z)}\left[\alpha(1-\alpha)+\alpha Z \frac{F^{\prime \prime}(Z)}{F^{\prime}(Z)}\right. \\
& \left.-\alpha(1-\alpha) Z \frac{F^{\prime}(Z)}{F(Z)}\right]+(1-\alpha) \frac{F^{\prime \prime}(Z)}{F^{\prime}(Z)}+2 \alpha \frac{F^{\prime}(Z)}{F(Z)}\end{aligned}$ \\
\hline $\begin{array}{l}a_{2} \neq-a_{1} \\
\text { II: } a_{4}=0 \\
a_{5} \neq 0\end{array}$ & $\begin{array}{l}Z=\left(v-c_{1}\right) \\
\times \exp \left[c_{2}\left(v+x-c_{1}\right)\right]\end{array}$ & $\begin{array}{l}v-c_{1}=\left(t-c_{3}\right) F(Z), c_{1}=\frac{a_{1}}{a_{5}} \\
\quad c_{2}=\frac{a_{5}}{a_{1}+a_{2}}, c_{3}=\frac{a_{3}}{a_{5}}\end{array}$ & $\begin{array}{l}\frac{c_{2} Z F(Z) F^{\prime \prime}(Z)}{F^{\prime}(Z)\left[F(Z)-Z F^{\prime}(Z)\right]}+\frac{c_{2}}{F(Z)}\left[F(Z)+Z F^{\prime}(Z)\right] \\
\quad=F(Z)\end{array}$ \\
\hline $\begin{array}{l}a_{2}=-a_{1} \\
a_{4}=0 \\
a_{5} \neq 0\end{array}$ & $Z=v+x$ & $\begin{array}{l}v-c_{1}=\left(t-c_{2}\right) F(Z), c_{1}=\frac{a_{1}}{a_{5}} \\
\quad c_{2}=\frac{a_{3}}{a_{5}}\end{array}$ & $F^{\prime \prime}(Z)-F(Z) F^{\prime}(Z)=0, F^{\prime}(Z) \neq 0$ \\
\hline $\mathrm{IV}: \quad \begin{array}{l}a_{3} \neq 0 \\
a_{4} \neq 0 \\
a_{5}=2 a_{4}\end{array}$ & $\begin{array}{l}Z=\left(v-c_{1}\right)(v+x \\
\left.\quad-c_{1}-c_{2}\right)\end{array}$ & $\begin{array}{c}v=c_{1}+e^{c_{3} t} F(Z), c_{1}=\frac{a_{1}}{a_{4}} \\
c_{2}=-\frac{2 a_{1}+a_{2}}{a_{4}} \\
c_{3}=-\frac{a_{4}}{a_{3}}\end{array}$ & $\frac{F^{\prime \prime}(Z) F(Z)}{F^{\prime}(Z)\left[F(Z)-Z F^{\prime}(Z)\right]}+\frac{2 F^{\prime}(Z)}{F(Z)}-c_{3}=0$ \\
\hline $\begin{array}{l}a_{3}=0 \\
\mathrm{~V}: \quad a_{4} \neq 0 \\
a_{5}=2 a_{4}\end{array}$ & $Z=t$ & $\begin{array}{c}\left(v-c_{1}\right)\left(v+x-c_{1}-c_{2}\right)=F(Z) \\
c_{1}=\frac{a_{1}}{a_{4}}, c_{2}=-\frac{2 a_{1}+a_{2}}{a_{4}}\end{array}$ & $F^{\prime}(Z)=-2$ \\
\hline $\begin{aligned} & a_{1} \neq 0 \\
\mathrm{VI}: & a_{4} \neq 0 \\
& a_{5}=a_{4}\end{aligned}$ & $\begin{aligned} Z & =c_{1} v \\
& -\log \left|v+x+c_{2}\right|\end{aligned}$ & $\begin{array}{c}e^{c_{1} v}=\left(t+c_{3}\right) F(Z), c_{1}=\frac{a_{4}}{a_{1}} \\
c_{2}=\frac{a_{1}+a_{2}}{a_{4}}, c_{3}=\frac{a_{3}}{a_{4}}\end{array}$ & $\begin{array}{l}c_{1} e^{Z}\left[\frac{F(Z) F^{\prime \prime}(Z)}{F^{\prime 2}(Z)}+\frac{F^{\prime}(Z)}{F(Z)}\left(1-\frac{F(Z)}{F^{\prime}(Z)}\right)^{2}-1\right] \\
=F(Z)\left(1-\frac{F(Z)}{F^{\prime}(Z)}\right), F^{\prime}(Z) \neq F(Z)\end{array}$ \\
\hline $\begin{array}{l}a_{1}=0 \\
\mathrm{VII}: \quad a_{4} \neq 0 \\
\\
a_{5}=a_{4}\end{array}$ & $Z=v$ & $\begin{array}{l}v=\left(t+c_{2}\right) F(Z)-x-c_{1} \\
\quad c_{1}=\frac{a_{2}}{a_{4}}, c_{2}=\frac{a_{3}}{a_{4}}\end{array}$ & $F^{\prime \prime}(Z)-F(Z) F^{\prime}(Z)=0, F^{\prime}(Z) \neq 0$ \\
\hline $\begin{array}{l}\text { VIII: } \\
\qquad \begin{array}{l}a_{1} a_{2} a_{3} \neq 0 \\
a_{2} \neq-a_{1}, \\
a_{4}=a_{5}=0\end{array}\end{array}$ & $Z=a_{2} v-a_{1} x$ & $a_{3} v=a_{1} t+F(Z)$ & $\begin{array}{l}a_{3}^{2} F^{\prime \prime}(Z)-\left(a_{1}+a_{2}\right) F^{\prime 2}(Z)+a_{3} F^{\prime}(Z)=0 \\
\quad F^{\prime}(Z) \neq=\frac{a_{3}}{a_{1}+a_{2}}, 0\end{array}$ \\
\hline $\begin{array}{c}a_{1}=a_{4} \\
\text { IX: } \quad=a_{5}=0, \\
a_{2} a_{3} \neq 0\end{array}$ & $Z=v$ & $a_{3} x=a_{2} t+F(Z)$ & $\begin{array}{l}a_{3} F^{\prime \prime}(Z)-a_{2} F^{\prime}(Z)-a_{2} a_{3}=0 \\
\quad F^{\prime}(Z) \neq=-a_{3}\end{array}$ \\
\hline $\mathrm{X}: \begin{array}{c}a_{2}=a_{4} \\
=a_{5}=0, \\
a_{1} a_{3} \neq 0\end{array}$ & $Z=x$ & $a_{3} v=a_{1} t+F(Z)$ & $\begin{array}{l}a_{3}^{2} F^{\prime \prime}(Z)-a_{1} F^{\prime 2}(Z)-a_{1} a_{3} F^{\prime}(Z)=0, \\
\quad F^{\prime}(Z) \neq-a_{3}, 0\end{array}$ \\
\hline
\end{tabular}

Various cases need to be considered that depend on the nature of the constants $a_{i}(i=1,2, \ldots, 5)$. The results are summarized in Table 1 .

In all other cases, the solutions are only of the from $v=c_{1} x+c_{2}$ with $c_{1}\left(c_{1}+1\right) \neq 0$ and hence are not considered. 


\subsection{Comparison of solutions of the potential equation (1.30) obtained from nonclassical symmetries and point symmetries}

We now show that the solutions (4.4a) include at least one nonclassical solution of the potential equation (1.30), i.e. we obtain a solution of (1.30) that does not arise as an invariant solution for any point symmetry admitted by (1.30). In particular, in (4.4a), let $b=2, \gamma=1, c=0, b_{1}=0, b_{2}=1$. Then we obtain the solution

$$
t=-\frac{1}{8}[\log |\sinh (3 v+2 x)|-\log (\cosh (v+2 x))]
$$

of the potential equation (1.30). The proof that the solution (4.6) is a nonclassical solution of the potential equation (1.30) (and hence a nonclassical potential solution of the given scalar PDE (1.28)) is given below. The proof involves showing that the solution (4.6) cannot be obtained from any of the invariant solutions found in $\S 4.2$.

\subsubsection{Comparison of (4.6) and Case I}

From Case I, we have

$$
x=Z\left(v-c_{1}\right)^{-\alpha}-v+c_{1}+c_{2}, \quad t=\frac{\left(v-c_{1}\right)^{1-\alpha}}{F(Z)}+c_{3}, \quad \alpha \neq 0,1 .
$$

The substitution of (4.7) into (4.6) yields

$$
\begin{aligned}
& \frac{8\left(v-c_{1}\right)^{1-\alpha}}{F(Z)}+8 c_{3}+\log \left|\sinh \left(v+2 Z\left(v-c_{1}\right)^{-\alpha}+2 c_{1}+2 c_{2}\right)\right| \\
& -\log \left[\cosh \left(v-2 Z\left(v-c_{1}\right)^{-\alpha}-2 c_{1}-2 c_{2}\right)\right]=0 .
\end{aligned}
$$

The invariant solution in Case I includes the solution (4.6) if and only if there exist some constants $\alpha, c_{1}, c_{2}, c_{3}$ such that equation (4.8) holds for all $v$ and $Z$. Two cases must be considered, depending on the sign of $\alpha-1$.

4.3.1.1 $\alpha<1$

Let $v \rightarrow+\infty$ in (4.8). Then the 1.h.s. of (4.8) $\frac{8 v^{1-\alpha}}{F(Z)}$ as $v \rightarrow+\infty$, which is impossible.

4.3.1.2 $\alpha>1$

Let $v \rightarrow+\infty$ in (4.8). Then the 1.h.s. of (4.8) $\sim 8 c_{3}$ as $v \rightarrow+\infty$, which leads to $c_{3}=0$. Thus (4.8) reduces to

$$
\frac{8}{F(Z)}+\frac{\log |\sinh (v+y)|-\log [\cosh (v-y)]}{\left(v-c_{1}\right)^{1-\alpha}}=0,
$$

where $y=2 Z\left(v-c_{1}\right)^{-\alpha}+2 c_{1}+2 c_{2}$, which must hold for some constants $\alpha, c_{1}, c_{2}$, for all $v$ and $Z$. Now let $v \rightarrow+\infty$ in (4.9). Then the 1.h.s. of (4.9) $\sim \frac{8}{F(Z)}$ as $v \rightarrow+\infty$, which is impossible.

The arguments to show that the other nine cases arising in Table 1 all yield similarity solutions distinct from (4.6) are similar to those for Case I. In Cases II-V, one compares the solutions as $v \rightarrow+\infty$. In Case VI, one does the comparison as $v \rightarrow-\infty$ if $c_{1}>0$; as $v \rightarrow+\infty$ if $c_{1}<0$. In Case VII, one compares the solutions as $x \rightarrow+\infty$. In Case VIII, for $c_{3} c_{4}\left(c_{3}-c_{4}\right)>0$, one first compares the solutions as $v \rightarrow+\infty$ and then compares them 
separately as $v \rightarrow-\infty$ and $x \rightarrow-\infty$; for $c_{3} c_{4}\left(c_{3}-c_{4}\right)<0$, one first compares the solutions as $v \rightarrow-\infty$ and then compares them separately as $v \rightarrow+\infty$ and $x \rightarrow+\infty$. In Case IX, one compares the solutions as $x \rightarrow+\infty$. Finally, in Case $\mathrm{X}$, one does the comparison as $x \rightarrow+\infty$ if $c_{3}>0$; as $x \rightarrow-\infty$ if $c_{3}<0$.

Thus, we have completed the proof that the solution (4.6) of the potential equation (1.30) is a nonclassical solution of (1.30) and a nonclassical potential solution of the given nonlinear heat conduction equation (1.28) with $K(u)=\frac{1}{u^{2}+u}$. In a similar manner, one can prove that the solutions (4.4b) include at least one nonclassical potential solution of the given nonlinear heat conduction equation (1.28) with $K(u)=\frac{1}{u^{2}+u}$.

\section{Concluding remarks and important extensions to other PDEs}

In this paper, we have used the nonlinear heat conduction equation as a prototypical example to show that if a given second order scalar PDE is in conservation law form, then one can systematically obtain new solutions by applying the Nonclassical Method to a related potential equation. Such solutions, called nonclassical potential solutions, cannot be obtained as invariant solutions of admitted point symmetries of either the given scalar PDE or the related potential equation, or through the Nonclassical Method $(\tau \equiv 1)$ applied to the given scalar PDE. We denote the Nonclassical Method $(\tau \equiv 1)$ applied to a related potential equation as the Nonclassical Potential Equation Method (NPEM).

\subsection{Important extensions to other types of PDEs}

One can extend the NPEM to a higher order PDE of the form

$$
\frac{\partial^{n} u}{\partial t^{n}}=\frac{D}{D x}\left(f\left(x, t, u, \partial u, \ldots, \partial^{N} u\right)\right),
$$

where $\partial^{k} u$ denotes the $k$ th order partial derivatives of $u$. Here it is easy to show that PDE (5.1) is equivalent to the related potential equation given by

$$
\frac{\partial^{n} w}{\partial t^{n}}=\left.\left[f\left(x, t, u, \partial u, \ldots, \partial^{N} u\right)\right]\right|_{u=w_{x}}
$$

for some potential variable $w$. Moreover, if $w=\phi(x, t)$ solves (5.2), then $u=\phi_{x}(x, t)$ solves (5.1).

More generally, if PDE (5.1) is of the form

$$
\frac{\partial^{n} u}{\partial t^{n}}=\frac{D^{m}}{D x^{m}}\left(f\left(x, t, u, \partial u, \ldots, \partial^{N} u\right)\right)+\sum_{k=1}^{m-1} \sum_{j=0}^{q_{k}} A_{k j}(t) \frac{\partial^{k+j} u}{\partial x^{k} \partial t^{j}},
$$

then one can show that it is also equivalent to each of the $m$ related potential equations

$$
\frac{\partial^{n} w}{\partial t^{n}}=\left.\left[\frac{D^{m-i}}{D x^{m-i}}\left(f\left(x, t, u, \partial u, \ldots, \partial^{N} u\right)\right)\right]\right|_{u=\frac{\partial^{i} w}{\partial x^{i}}}+\sum_{k=1}^{m-1} \sum_{j=0}^{q_{k}} A_{k j}(t) \frac{\partial^{k+j} w}{\partial x^{k} \partial t^{j}},
$$

$i=1,2, \ldots, m$. Moreover, if $w=\phi_{i}(x, t)$ solves (5.4), then $u=\frac{\partial^{i} \phi_{i}(x, t)}{\partial x^{i}}$ solves (5.3), $i=1,2, \ldots, m$. 
As a first example, consider the nonlinear heat conduction equation (1.28), which can be written in the form

$$
\frac{\partial u}{\partial t}=\frac{\partial^{2}}{\partial x^{2}}(L(u))=\frac{\partial}{\partial x}\left(K(u) u_{x}\right)
$$

where $L(u)=\int^{u} K(s) d s$. Equation (5.5) yields potential systems

$$
\begin{gathered}
v_{x}=u, \\
v_{t}=\frac{\partial}{\partial x}(L(u))=K(u) u_{x}
\end{gathered}
$$

and

$$
\begin{aligned}
v_{x} & =u, \\
w_{x} & =v, \\
w_{t} & =L(u) .
\end{aligned}
$$

The substitution of (5.6a) into (5.6b) yields the first equivalent potential equation

$$
v_{t}=K\left(v_{x}\right) v_{x x}=L^{\prime}\left(v_{x}\right) v_{x x}
$$

If $v=\phi(x, t)$ solves the potential equation (5.8), then $u=\phi_{x}(x, t)$ solves the nonlinear heat conduction equation (5.5).

The substitution of (5.7b) into (5.7a) yields $u=w_{x x}$. Hence from (5.7c), we obtain the second equivalent potential equation [3]

$$
w_{t}=L\left(w_{x x}\right)
$$

If $w=\Phi(x, t)$ solves the potential equation (5.9), then $u=\Phi_{x x}(x, t)$ solves the nonlinear heat conduction equation (5.5).

As a second example, consider the Boussinesq equation

$$
u_{t t}=a u_{x x}+b\left(u^{2}\right)_{x x}+c u_{x x x x}=\frac{D^{2}}{D x^{2}}\left(a u+b u^{2}+c u_{x x}\right), \quad a, b, c=\text { const. }
$$

Equation (5.10) yields the equivalent potential systems

$$
\begin{gathered}
v_{x}=u_{t} \\
v_{t}=\frac{D}{D x}\left(a u+b u^{2}+c u_{x x}\right)
\end{gathered}
$$

and

$$
\begin{gathered}
w_{t}=v, \\
w_{x}=u, \\
v_{t}=\frac{D}{D x}\left(a u+b u^{2}+c u_{x x}\right) .
\end{gathered}
$$


The substitution of $(5.12 \mathrm{a}, \mathrm{b})$ into $(5.12 \mathrm{c})$ yields the first equivalent potential equation

$$
w_{t t}=a w_{x x}+2 b w_{x} w_{x x}+c w_{x x x x}=\frac{D}{D x}\left(a w_{x}+b w_{x}^{2}+c w_{x x x}\right) .
$$

If $w=\phi(x, t)$ solves the potential equation (5.13), then $u=\phi_{x}(x, t)$ solves the Boussinesq equation (5.10).

In turn, the first potential equation (5.13) is equivalent to the potential systems

$$
\begin{gathered}
Z_{x}^{(1)}=w_{t}, \\
Z_{t}^{(1)}=a w_{x}+b w_{x}^{2}+c w_{x x x}
\end{gathered}
$$

and

$$
\begin{gathered}
Z_{t}^{(2)}=Z^{(1)}, \\
Z_{x}^{(2)}=w, \\
Z_{t}^{(1)}=a w_{x}+b w_{x}^{2}+c w_{x x x} .
\end{gathered}
$$

The substitution of $(5.15 \mathrm{a}, \mathrm{b})$ into $(5.15 \mathrm{c})$ yields the second equivalent potential equation

$$
Z_{t t}=a Z_{x x}+b\left(Z_{x x}\right)^{2}+c Z_{x x x x}
$$

with $Z=Z^{(2)}$. If $Z=\Phi(x, t)$ solves the potential equation (5.16), then $u=\Phi_{x x}(x, t)$ solves the Boussinesq equation (5.10).

Most importantly, any method (numerical, perturbation, or qualitative), in addition to analytical methods such as the Classical Method and Nonclassical Method, applied to any one of the $m$ related potential equations (5.4) could yield new results for a given scalar PDE (5.3) and, mutatis mutandis, for any one of the other related potential equations.

We further remark that one could extend our work to $n$-dimensional systems of PDEs with at least one PDE in conservation law form.

\subsection{Further remarks}

(1) Consider the PDE in conservation law form

$$
\frac{\partial u}{\partial t}=\frac{D}{D x} f\left(x, t, u, \partial u, \ldots, \partial^{N} u\right)
$$

It is important to note that a nonclassical symmetry of the form

$$
Y=\xi(x, t) \frac{\partial}{\partial x}+\frac{\partial}{\partial t}+\zeta(x, t) \frac{\partial}{\partial v}
$$

admitted by the related potential equation

$$
v_{t}=\left.\left[f\left(x, t, u, \partial u, \ldots, \partial^{N} u\right)\right]\right|_{u=v_{x}}
$$

could yield nonclassical potential solutions of (5.17). 
As an example, consider the nonclassical symmetry (3.14a) in Section 3.3 with $\gamma=\frac{1}{4}$ so that it becomes $Y_{1}=b \tanh \left(\frac{b}{4} x+c\right) \frac{\partial}{\partial x}+\frac{\partial}{\partial t}-\frac{1}{2} b \tanh \left(\frac{b}{4} x+c\right) \frac{\partial}{\partial v}$. One can show that this nonclassical symmetry yields the nonclassical potential solution

$$
u=-\frac{1}{2}+\frac{1}{2} \cosh \left(\frac{1}{4} b x+c\right)\left[c_{1} \exp \left(\frac{1}{2} b^{2} t\right)+\sinh ^{2}\left(\frac{1}{4} b x+c\right)\right]^{-1 / 2},
$$

with the arbitrary constant $c_{1} \neq 0$, of the nonlinear heat conduction equation (1.28) with $K(u)=\frac{1}{u^{2}+u}$.

In Gandarias [12], the solution

$$
u=\frac{k_{1}}{k}\left[\tanh \left(k_{1}(x-k t)\right)-\tanh \left(k_{1}(x+k t)\right)\right]
$$

of the nonlinear heat conduction equation

$$
u_{t}=\left(u^{-1} u_{x}\right)_{x}
$$

was obtained by a procedure which is equivalent to finding a nonclassical solution of the related potential equation

$$
v_{t}=\frac{v_{x x}}{v_{x}}
$$

from its admitted nonclassical symmetry

$$
Y=k \frac{\partial}{\partial x}+\frac{\partial}{\partial t}-2 k_{1} \tanh \left[k_{1}\left(x+k t+k_{2}\right)\right] \frac{\partial}{\partial v} .
$$

The nonclassical symmetry (5.24) results from Case II in $\$ 2.1$ (equation (2.6) for $K(u)$ ), which we have not considered further in this paper.

(2) One can show that the purported 'nonclassical potential symmetries'

$$
Y_{1}=k \frac{\partial}{\partial x}+\frac{\partial}{\partial t}+2 \tan \left(v+k t+k_{1}\right) \frac{\partial}{\partial v}
$$

and

$$
Y_{2}=k \frac{\partial}{\partial x}+\frac{\partial}{\partial t}+2 \tan \left(x+v+k_{1}\right) \frac{\partial}{\partial v}
$$

of the nonlinear heat equation (5.22), presented in Gandarias [12], are not nonclassical symmetries of the potential equation (5.23) (and hence are not nonclassical potential symmetries of the PDE (5.22)). Moreover, the corresponding displayed 'solutions' in Gandarias [12] of the potential equation (5.23) are not solutions of (5.23).

(3) From our work exhibited in $\S 3.3$, one can easily see that the potential equation (5.23) admits the nonclassical symmetries

$$
\begin{aligned}
& Y_{1}=b \tanh \left(-\frac{1}{2} b v+b \gamma x+c\right) \frac{\partial}{\partial x}+\frac{\partial}{\partial t}-2 \gamma b \tanh \left(-\frac{1}{2} b v+b \gamma x+c\right) \frac{\partial}{\partial v} \\
& Y_{2}=b \operatorname{coth}\left(-\frac{1}{2} b v+b \gamma x+c\right) \frac{\partial}{\partial x}+\frac{\partial}{\partial t}-2 \gamma b \operatorname{coth}\left(-\frac{1}{2} b v+b \gamma x+c\right) \frac{\partial}{\partial v}
\end{aligned}
$$


with $b \neq 0$ and $\gamma \neq 0$. Correspondingly, one can find nonclassical potential solutions of the nonlinear heat conduction equation (5.22) that arise from its nonclassical potential symmetries $(5.26 \mathrm{a}, \mathrm{b})$.

(4) For $\tau \equiv 1$, the Nonclassical Method is much easier to apply to a potential equation than to a potential system, unlike the situation when seeking point symmetries for a potential equation vis-a-vis a potential system.

(5) For $K(u)=\frac{1}{u^{2}+u}$, the invariant solutions in Cases I-VII in Table I for the admitted point symmetries (3.3) of the potential equation (1.30) when $a_{5} \neq 0$, are new and in particular cannot be obtained from the invariance of (1.28) under point symmetries.

(6) By complexifying the parameters in (4.4a), one can obtain the following family of quasi-periodic solutions of the potential equation (1.30) for $K\left(v_{x}\right)=\frac{1}{v_{x}^{2}+v_{x}}$ :

$\sin \left[b\left(2 \gamma+\frac{1}{2}\right) v+b \gamma x+c\right]=e^{b^{2}\left(4 \gamma^{2}+2 \gamma\right) t}\left[c_{1} \sin \left(\frac{1}{2} b v-b \gamma x\right)+c_{2} \cos \left(\frac{1}{2} b v-b \gamma x\right)\right]$,

where $b, c, \gamma, c_{1}, c_{2}$ are arbitrary real constants with $b \neq 0, \gamma \neq 0,-\frac{1}{2}$ and $c_{1}^{2}+c_{2}^{2} \neq 0$.

(7) From our work in $\S 2.1 .1$, we see that if the nonlinear heat conduction (1.28) has a nonclassical potential solution related to its potential equation (1.30), then it is necessary that the conductivity $K(u)$ satisfy the first order Bernoulli equation

$$
\begin{aligned}
& \left(A_{1} u^{3}+A_{2} u^{2}+A_{3} u+A_{4}\right) K^{\prime}(u)+\left(B_{1} u^{2}+B_{2} u+B_{3}\right) K(u) \\
& \quad+\left(C_{1} u^{3}+C_{2} u^{2}+C_{3} u+C_{4}\right) K^{2}(u)=0
\end{aligned}
$$

for some constants $A_{i}, B_{j}, C_{k}$.

(8) Asymptotic analysis seems to be an effective tool for comparing solutions.

\section{Acknowledgements}

The authors thank the referees for helpful remarks. G. Bluman acknowledges the financial support of NSERC of Canada. Z. Yan acknowledges the financial support of a PIMS PDF, the NNSF of China (No. 10401039) and the NKBRSF of China (No. 2004CB318000).

\section{References}

[1] Akhatov, I. S., Gazizov, R. K. \& Ibragimov, N. H. (1987) Group classification of the equations of nonlinear filtration. Sov. Math. Dokl. 35, 384-386.

[2] Arrigo, D. J. \& Hill, J. M. (1995) Nonclassical symmetries for nonlinear diffusion and absorption. Stud. Appl. Math. 72, 21-39.

[3] Bluman, G. W. (1993) Use and construction of potential symmetries. Math. Comput. Modelling, 18, 1-14.

[4] Bluman, G. W. \& Anco, S. C. (2002) Symmetry and Integration Methods for Differential Equations. Springer.

[5] Bluman, G. W. \& Cole, J. D. (1969) The general similarity solution of the heat equation. J. Math. Mech. 18, 1025-1042.

[6] Bluman, G. W. \& Cole, J. D. (1974) Similarity Methods for Differential Equations. SpringerVerlag. 
[7] Bluman, G. W. \& Kumei, S. (1989) Symmetries and Differential Equations. Springer-Verlag.

[8] Bluman, G. W., Kumei, S. \& ReID, G. J. (1988) New classes of symmetries for partial differential equations. J. Math. Phys. 29, 806-811. Erratum: J. Math. Phys. 29, 2320 (1988).

[9] Bluman, G. W. \& Shtelen, V. (1996) Developments in similarity methods related to pioneering work of Julian Cole. In: Mathematics is For Solving Problems, Cook, S. L. P., Roytburd, V. and Tulin, M. (eds.), SIAM, pp.105-118.

[10] Clarkson, P. A. \& Mansfield, E. L. (1994) Algorithms for the nonclassical method of symmetry reductions. SIAM J. Appl. Math. 54, 1693-1719.

[11] Clarkson, P. A. \& Priestley, T. J. (1998) Shallow water wave systems. Stud. Appl. Math. 101, 389-432.

[12] Gandarias, M. L. (2001) New symmetries for a model of fast diffusion. Phys. Lett. A, 286, 153-160.

[13] Gandarias, M. L. \& Bruzón, M. S. (2000) Symmetry analysis and solutions for a family of Cahn-Hilliard equations. Rep. Math. Phys. 46, 89-97.

[14] LeVI, D. \& Winternitz, P. (1989) Non-classical symmetry reduction: example of the Boussinesq equation. J. Phys. A, 22, 2915-2924.

[15] Mansfield, E. L., Reid, G. J. \& Clarkson, P. A. (1998) Nonclassical reductions of a 3+1cubic nonlinear Schrödinger system. Comp. Phys. Comm. 115, 460-488.

[16] Olver, P. J. (1986) Application of Lie Groups to Differential Equations. Spring-Verlag.

[17] Ovsiannikov, L. V. (1982) Group Analysis of Differential Equations. Academic Press.

[18] Saccomandi, G. (1997) Potential symmetries and direct reduction methods of order two. J. Phys. A, 30, 2211-2217. 